\title{
A class of nonmonotone trust region algorithm for solving unconstrained nonlinear optimization problems
}

\author{
Fulan Ye, Yang You, Zhen Chen, Baoguo Chen* \\ Research Centre for Science Technology and Society, \\ Fuzhou University of International Studies and Trade, Fuzhou 350202, China \\ *Corresponding author, e-mail: chenbg123@163.com
}

Received 10 Jun 2017

Accepted 15 Oct 2017

\begin{abstract}
Based on the nonmonotone line search technique proposed by $\mathrm{Gu}$ and Mo a nonmonotone trust region algorithm is proposed for solving unconstrained nonlinear optimization problems. The new algorithm is resets the ratio $\rho_{k}$ for evaluating whether the trial step $d_{k}$ is acceptable. The global and superlinear convergence of the algorithm are proved under suitable conditions. Numerical results show that the new algorithm is effective.
\end{abstract}

KEYWORDS: global convergence, superlinear convergence, numerical experiment

MSC2010: 90C30

\section{INTRODUCTION}

We consider the unconstrained nonlinear optimization problem

$$
\min _{x \in \mathbb{R}^{n}} f(x),
$$

where $f: \mathbb{R}^{n} \rightarrow \mathbb{R}$ is a continuously differentiable nonlinear function. The line search method and trust region method are the two principal methods for solving (1). The line search method produces a sequence $x_{0}, x_{1}, \ldots$, where $x_{k+1}$ is generated from the current approximate solution $x_{k}$, and the specific direction $d_{k}$ and a step size $\alpha_{k}>0$ by the rule $x_{k+1}=x_{k}+\alpha_{k} d_{k}$. The trust region methods obtain a trial step $d_{k}$ by solving the quadric program subproblem

$$
\min _{d \in \mathbb{R}^{n}} \phi_{k}(d)=g_{k}^{\mathrm{T}} d+\frac{1}{2} d^{\mathrm{T}} B_{k} d \text { s.t. }\|d\| \leqslant \Delta_{k},
$$

where $g_{k}=\nabla f\left(x_{k}\right), B_{k} \in \mathbb{R}^{n \times n}$ is a symmetric matrix which is either the exact Hessian matrix of $f$ at $x_{k}$ or an approximation for it, $\Delta_{k}>0$ is the trust region radius, and $\|\cdot\|$ denotes the Euclidean norm. The traditional trust region methods evaluate the trial step $d_{k}$ by the ratio

$$
\rho_{k}=\frac{f\left(x_{k}\right)-f\left(x_{k}+d_{k}\right)}{\phi_{k}(0)-\phi_{k}\left(d_{k}\right)} .
$$

The trial step $d_{k}$ is accepted whenever $\rho_{k}$ is greater than a positive constant $\mu$. Then we can set the new point $x_{k+1}=x_{k}+d_{k}$ and enlarge the trust region radius. Otherwise, the traditional trust region methods resolve the subproblem (2) by reducing the trust region radius until an acceptable step is found. Solving the region subproblems may lead us to solve one or more quadric program problems and increase the total cost of computation for large scale problems. Compared with line search techniques, new trust region methods have a strong convergence property, and a much lower computational cost than the traditional trust region methods ${ }^{1}$. Some theoretical and numerical results of these trust region methods with line search are also interesting. However, all these methods enforce a monotonic decrease in the objective function values at each iteration and this may slow the convergence rate in the minimization process ${ }^{2}$. To overcome the shortcomings, the earliest nonmonotone line search framework was developed by Grippo et $\mathrm{al}^{3}$ for Newton's method. In their approach, parameters $\lambda_{1}, \lambda_{2}, \sigma$ and $\beta$ are introduced, where $0<\lambda_{1}<\lambda_{2}, \beta, \sigma \in(0,1)$ and $\alpha_{k}=\bar{\alpha}_{k} \sigma^{h_{k}}$, where $\bar{\alpha}_{k} \in\left(\lambda_{1}, \lambda_{2}\right)$ is the trial step and $h_{k}$ is the smallest nonnegative integer such that

$$
f\left(x_{k}+d_{k}\right) \leqslant \max _{0 \leqslant j \leqslant m_{k}} f\left(x_{k-j}\right)+\beta \alpha_{k} \nabla f\left(x_{k}\right)^{\mathrm{T}} d_{k},
$$

the memory variable $m_{k}$ is a nondecreasing integer by setting

$$
m_{k}= \begin{cases}0, & k=0 \\ 0<m_{k} \leqslant \min \left\{m_{k-1}+1, M\right\}, & k>0\end{cases}
$$


where $M$ is a prefixed nonnegative integer. From then on, studies in nonlinear optimization have paid great attentions to it ${ }^{4,5}$. Deng et $\mathrm{al}^{2}$ made some changes in the common ratio (3) by resetting the rule as follows:

$$
\hat{\rho}_{k}=\frac{\max _{0 \leqslant j \leqslant m_{k}} f\left(x_{k-j}\right)-f\left(x_{k}+d_{k}\right)}{\phi_{k}(0)-\phi_{k}\left(d_{k}\right)} .
$$

The ratio (5) which assesses the agreement between the quadratic model and the objective function in trust region methods is the most common nonmonotone ratio. Some researchers showed that the nonmonotone method can improve both the possibility of finding the global optimum and the rate of convergence when a monotone scheme is forced to creep along the bottom of a narrow curved valley $^{6,7}$.

Although the nonmonotone technique (4) has many advantages, Zhang and Hager ${ }^{8}$ proposed a new nonmonotone line search algorithm, which had the same general form as the scheme of Grippo et $\mathrm{al}^{3}$ except that their 'max' was replaced by an average of function values. The nonmonotone line search found a step length $\beta$ to satisfy the inequality

$$
f\left(x_{k}+\beta d_{k}\right) \leqslant C_{k}+\delta \beta \nabla f\left(x_{k}\right)^{\mathrm{T}} d_{k},
$$

where

$$
C_{k}= \begin{cases}f\left(x_{k}\right), & k=0, \\ \left(\eta_{k-1} Q_{k-1} C_{k-1}+f\left(x_{k}\right)\right) / Q_{k}, & k \geqslant 1,\end{cases}
$$

and

$$
Q_{k}= \begin{cases}1, & k=0, \\ \eta_{k-1} Q_{k-1}+1, & k \geqslant 1,\end{cases}
$$

$\eta_{k-1} \in\left[\eta_{\min }, \eta_{\max }\right]$, where $\eta_{\min } \in[0,1)$ and $\eta_{\max } \in$ $\left[\eta_{\min }, 1\right]$ are two chosen parameters. Numerical results showed that the new nonmonotone method can improve the efficiency of the nonmonotone trust region methods.

Observe that $C_{k+1}$ is a convex combination of $C_{k}$ and $f\left(x_{k+1}\right)$. Since $C_{0}=f\left(x_{0}\right)$, we see that $C_{k}$ is a convex combination of the function values $f\left(x_{0}\right), f\left(x_{1}\right), \ldots, f\left(x_{k}\right)$. From (7), the degree of nonmonotonicity and (8) depend on the choice $\eta_{k}$. If $\eta_{k}=0$ for each $k$, then the line search is the usual Armijo line search. If $\eta_{k}=1$ for each $k$, then $C_{k}=A_{k}$, where

$$
A_{k}=\frac{1}{k+1} \sum_{i=0}^{k} f_{i}, \quad f_{i}=f\left(x_{i}\right),
$$

is the average function value. However, it becomes an encumbrance to update $\eta_{k}$ and $Q_{k}$ at each $k$ in practice. Recently $\mathrm{Gu}$ and $\mathrm{Mo}^{9}$ developed an algorithm that combines a new nonmonotone technique and trust region method for unconstrained optimization problems. The new nonmonotone line search is as follows:

$$
f\left(x_{k}+\beta d_{k}\right) \leqslant D_{k}+\delta \beta \nabla f\left(x_{k}\right)^{\mathrm{T}} d_{k},
$$

where the parameter $\eta \in(0,1)$ or a variable $\eta_{k}$ and

$$
D_{k}= \begin{cases}f\left(x_{k}\right), & k=0, \\ \eta D_{k-1}+(1-\eta) f\left(x_{k}\right), & k \geqslant 1,\end{cases}
$$

is a simple convex combination of the previous $D_{k-1}$ and $f_{k}$.

In this paper, we develop an algorithm which resets the ratio $\rho_{k}$ in the trust region method for unconstrained optimization problems. The algorithm does not restrict one to having a monotonic decrease in the objective function values at each iteration. Under suitable assumptions, we establish the global and superlinear convergence of the new algorithm. Numerical experiments show that our algorithm is quite effective.

\section{NEW NONMONOTONE TRUST REGION ALGORITHM}

For convenience, we denote $f\left(x_{k}\right)$ by $f_{k}$ and $g\left(x_{k}\right)$ by $g_{k}$, where $g\left(x_{k}\right) \in \mathbb{R}^{n}$ is the gradient of $f$ evaluated at $x_{k}$. The trial step $d_{k}$ is obtained by solving (2) at each iteration. We solve (2) such that $\left\|d_{k}\right\| \leqslant$ $\Delta_{k}$ and

$\phi_{k}(0)-\phi_{k}\left(d_{k}\right) \geqslant \tau\left\|g_{k}\right\| \min \left\{\Delta_{k},\left\|g_{k}\right\| /\left\|B_{k}\right\|\right\}$,

where $\tau \in(0,1)$ is a constant. Clearly if $B_{k}$ is a symmetric and positive definite diagonal matrix, we can obtain the solution $d_{k}$ easily. More precisely, if $\left\|B_{k}^{-1} g_{k}\right\| \leqslant \Delta_{k}$, then $d_{k}=-B_{k}^{-1} g_{k}$ is the optimal solution of (2); otherwise, if $\left\|B_{k}^{-1} g_{k}\right\|>\Delta_{k}$, we choose the optimal solution

$$
d_{k}=-\frac{\Delta_{k}}{\left\|B_{k}^{-1} g_{k}\right\|} B_{k}^{-1} g_{k} .
$$

To decide whether the obtained trial step $d_{k}$ will be accepted or not, and how to adjust the new trust region radius, we compute the ratio $\rho_{k}$ between the actual reduction, $D_{k}-f\left(x_{k}+d_{k}\right)$, and the predicted reduction, $\phi_{k}(0)-\phi_{k}\left(d_{k}\right)$, as follows:

$$
\rho_{k}=\frac{D_{k}-f\left(x_{k}+d_{k}\right)}{\phi_{k}(0)-\phi_{k}\left(d_{k}\right)},
$$

where $D_{k}$ is computed from (10). If $\rho_{k} \geqslant \mu$, where $\mu \in(0,1)$ is a constant, we accept the trial step $d_{k}$, 
set $x_{k+1}=x_{k}+d_{k}$ and enlarge the trust region radius $\Delta_{k}$. Otherwise we set $x_{k+1}=x_{k}$, reduce the trust region radius, and re-solve (2).

We now propose the following new nonmonotone trust region algorithm.

\section{Algorithm 1}

Step 1: Choose parameters $\eta \in(0,1), \mu \in(0,1)$, $\Delta_{0}>0,0<c_{1}<1<c_{2}$. Given an arbitrary point $x_{0} \in \mathbb{R}^{n}$ and a symmetric matrix $B_{0} \in$ $\mathbb{R}^{n \times n}$. Set $k:=0$.

Step 2: Compute $g_{k}$. If $\left\|g_{k}\right\|=0$, stop. Otherwise, go to Step 2.

Step 3: Compute an approximate solution $d_{k}$ so that $\left\|d_{k}\right\| \leqslant \Delta_{k}$ and (11) is satisfied.

Step 4: Compute $D_{k}$ by (10), and $\rho_{k}$ by (12).

Step 5: Set

$$
x_{k+1}= \begin{cases}x_{k}+d_{k}, & \rho_{k} \geqslant \mu, \\ x_{k}, & \text { otherwise. }\end{cases}
$$

Step 6: Compute $\Delta_{k+1}$ as

$$
\Delta_{k+1}= \begin{cases}c_{1}\left\|d_{k}\right\| & \text { if } \rho_{k}<\mu, \\ c_{2}\left\|d_{k}\right\| & \text { if } \rho_{k} \geqslant \mu .\end{cases}
$$

Step 7: Update $B_{k}$. Set $k:=k+1$ and go to Step 2 .

\section{GLOBAL CONVERGENCE}

In this section, we discuss the global convergence of Algorithm 1. Suppose an infinite sequence of iterations $\left\{x_{k}\right\}$ is obtained from Algorithm 1. Some common assumptions are as follows:

$\left(\mathrm{A}_{1}\right)$ The level set $\Omega_{0}=\left\{x \in \mathbb{R}^{n} \mid f(x) \leqslant f\left(x_{0}\right)\right\}$ is bounded.

$\left(\mathrm{A}_{2}\right)$ The gradient function of $g(x)$ is Lipschitz continuous in $\Omega_{0}$.

$\left(\mathrm{A}_{3}\right)$ The matrix sequence $\left\{B_{k}\right\}$ is uniformly bounded. lows:

For simplicity, we define two index sets as fol-

$$
I=\left\{k \mid \rho_{k} \geqslant \mu\right\}, J=\left\{k \mid \rho_{k}<\mu\right\} .
$$

Lemma 1 Suppose that the sequence $\left\{x_{k}\right\}$ is generated by Algorithm 1. Then the following inequality holds for all $k$ :

$$
f_{k+1} \leqslant D_{k+1} \leqslant D_{k} .
$$

Proof: Firstly we prove that (15) holds for all $k \in I$, i.e.,

$$
f_{k+1} \leqslant D_{k+1} \leqslant D_{k} \quad \forall k \in I .
$$

For $k \in I$, according to $\rho_{k} \geqslant \mu$, (11) and (12), we know that

$$
f_{k+1} \leqslant D_{k}-\mu \tau\left\|g_{k}\right\| \min \left\{\Delta_{k},\left\|g_{k}\right\| /\left\|B_{k}\right\|\right\} .
$$

From (10) and (17), we obtain

$$
\begin{aligned}
D_{k+1}= & \eta D_{k}+(1-\eta) f\left(x_{k+1}\right) \\
\leqslant & \eta D_{k}+(1-\eta) D_{k} \\
& \quad-\mu \tau\left\|g_{k}\right\| \min \left\{\Delta_{k},\left\|g_{k}\right\| /\left\|B_{k}\right\|\right\} \\
= & D_{k}-\mu \tau\left\|g_{k}\right\| \min \left\{\Delta_{k},\left\|g_{k}\right\| /\left\|B_{k}\right\|\right\} .
\end{aligned}
$$

By (10), if $\eta \neq 0$, we have

$$
D_{k+1}-D_{k}=\frac{(1-\eta)\left(f_{k+1}-D_{k+1}\right)}{\eta},
$$

and if $\eta=0$, we have

$$
D_{k+1}=f_{k+1} .
$$

Combining (18)-(20), it follows that (16) holds.

Secondly, we prove that (15) holds for all $k \in J$. From Step 4 of Algorithm 1, we obtain $x_{k+1}=x_{k}$ and $f_{k+1}=f_{k}$ for all $k \in J$. First we prove that $f_{k+1} \leqslant$ $D_{k+1}$. We consider two cases, respectively.

(i) If $k-1 \in I$. According to (16), we have $f_{k} \leqslant$ $D_{k}$. Following from (10) and $f_{k+1}=f_{k}$, we can deduce that

$$
\begin{aligned}
D_{k+1} & =\eta D_{k}+(1-\eta) f_{k+1} \\
& \geqslant \eta f_{k+1}+(1-\eta) f_{k+1}=f_{k+1} .
\end{aligned}
$$

(ii) If $k-1 \in J$. In this case, we define an index set $\mathscr{F}=\{i \mid 1<i \leqslant k, k-i \in I\}$. If $\mathscr{F}=\varnothing$, by Step 4 of Algorithm 1 we obtain $f_{0}=f_{k-j}=f_{k+1}, j=0$, $1, \ldots, k-1$. From (10) we obtain

$$
D_{k+1}=D_{k}=f_{k+1} .
$$

We now suppose that $\mathscr{F} \neq \varnothing$. Let $s=\min \{i: i \in$ $\mathscr{F}\}$. Then we have

$$
f_{k+1}=f_{k}=f_{k-j}, j=0,1, \ldots, s-1 .
$$

By (10) we have

$$
D_{k}=\eta D_{k-1}+(1-\eta) f_{k}, k \geqslant 1 .
$$

Using (24) repeatedly we obtain

$$
\begin{aligned}
& \eta D_{k}+(1-\eta) f_{k+1}=\eta^{s} D_{k-s+1} \\
& \quad+\sum_{i=0}^{s-2} \eta^{i+1}(1-\eta) f_{k-i}+(1-\eta) f_{k+1}
\end{aligned}
$$


According to the definition of $\mathscr{F}, s$ and (16), we have $k-s \in I$ and $D_{k-s+1} \geqslant f_{k-s+1}$. Combining (23) and (25) we deduce that

$$
\begin{aligned}
\eta D_{k} & +(1-\eta) f_{k+1} \\
& \geqslant \eta^{s} f_{k-s+1}+\sum_{i=0}^{s-2} \eta^{i+1}(1-\eta) f_{k-i}+(1-\eta) f_{k+1} \\
& =\left[\eta^{s}+\sum_{i=0}^{s-2} \eta^{i+1}(1-\eta)+(1-\eta)\right] f_{k+1} \\
& =f_{k+1}
\end{aligned}
$$

Hence it follows from (10) and (26) that

$$
D_{k+1}=\eta D_{k}+(1-\eta) f_{k+1} \geqslant f_{k+1} .
$$

By (21), (22) and (27), we conclude that

$$
f_{k+1} \leqslant D_{k+1}, \quad \forall k \in J .
$$

If $\eta \neq 0$, by (19) and (28) we obtain that $f_{k+1} \leqslant$ $D_{k+1} \leqslant D_{k}$. If $\eta=0$, then, by (10) and $k \in J$, we obtain $D_{k+1}=f_{k+1}=f_{k}$. Combining $k-1 \in J$ and (28), we obtain that $f_{k} \leqslant D_{k}$. Thus (15) holds for all $k \in J$.

Lemma 2 Suppose that $\mathrm{A}_{1}$ holds. Then the sequence $\left\{x_{k}\right\}$ generated by Algorithm 1 is contained in the level set $\Omega_{0}$.

Proof: From Lemma $1, \mathrm{~A}_{1}$ and $D_{0}=f_{0}$, we can easily obtain the assertion.

For convenience, we say an iteration point is successful if $x_{k+1}=x_{k}+d_{k}$, and unsuccessful if $x_{k+1}=x_{k}$.

Lemma 3 (Ref. 10) Suppose that $\mathrm{A}_{2}$ and $\mathrm{A}_{3}$ hold, the sequence $\left\{x_{k}\right\}$ is generated by Algorithm 1, and the following inequality holds for all $k$ :

$$
\left\|g_{k}\right\| \geqslant \epsilon,
$$

where $\epsilon \in(0,1)$ is a constant. Then for each $k$, there is a nonnegative integer $m$ such that $x_{k+m+1}$ is a successful iteration point.

Based on the above lemmas, we establish the global convergence of Algorithm 1.

Theorem 1 Suppose that $\mathrm{A}_{1}-\mathrm{A}_{3}$ hold. Then the sequence $\left\{x_{k}\right\}$ generated by Algorithm 1 satisfies

$$
\liminf _{k \rightarrow \infty}\left\|g_{k}\right\|=0
$$

Proof: By contradiction, we suppose that there exists a constant $\epsilon \in(0,1)$ such that the following inequality holds for all $k$ :

$$
\left\|g_{k}\right\| \geqslant \epsilon .
$$

Firstly, we prove that

$$
\lim _{k \rightarrow \infty, k \in I} \Delta_{k}=0 .
$$

From the proof of Lemma 3 in Ref. 10, we know that $I$ is an infinite set. If $k \in I$, then by (18) and (31), we obtain

$$
D_{k+1} \leqslant D_{k}-\mu \tau \epsilon(1-\eta) \min \left\{\Delta_{k}, \epsilon /\left\|B_{k}\right\|\right\} .
$$

From Lemma 1, we know that $\left\{D_{k}\right\}$ is nonincreasing and $f_{k+1} \leqslant D_{k+1}$ for all $k \geqslant 0$. By $\mathrm{A}_{1}$, Lemma 2 and the continuity of $f$, we know that the sequence $\left\{f_{k}\right\}$ is bounded below, and $\left\{D_{k}\right\}$ is convergent. By taking limits as $k \rightarrow \infty$ and $k \in I$ in (33), we have

$$
\lim _{k \rightarrow \infty, k \in I} \min \left\{\Delta_{k}, \epsilon /\left\|B_{k}\right\|\right\}=0 .
$$

By $\mathrm{A}_{3}$ and (34) we see that (32) holds.

Next, we prove that

$$
\lim _{k \rightarrow \infty} \Delta_{k}=0 .
$$

(i) If $J$ is a finite set, then (32) holds, which implies that (35) holds.

(ii) If $J$ is an infinite set, we define $\mathscr{F}_{1}=\left\{i_{k} \mid k=\right.$ $0,1, \ldots\}$ which is a subset of $J$ satisfying

$$
i_{1}=\min \{j \mid j \in J\},
$$

and

$i_{k+1}=\min \left\{j \in J \mid j-1 \in I, j-1>i_{k}\right\}, \quad \forall k \geqslant 1$.

According to Lemma 3, we know that $\mathscr{F}_{1}$ is an infinite set. For $k \geqslant 1$, by the definition of $i_{k}$ we know that $i_{k}-1 \in I$. According to Step 5 of Algorithm 1, we have

$$
\Delta_{i_{k}} \leqslant c_{2} \Delta_{i_{k}-1} .
$$

The definition of $i_{k+1}$ implies that there exists at least one integer $l$ such that

$$
i_{k}+l<i_{k+1}-1, \quad i_{k}+l \in J .
$$

Let $l_{k}$ be the maximum integer satisfying (37). It follows from Step 5 of Algorithm 1 that

$$
\Delta_{i_{k}+l} \geqslant \Delta_{i_{k}+l+1}, \quad l=0,1, \ldots, l_{k},
$$


and

$\Delta_{i_{k}+l} \leqslant \Delta_{i_{k}+l+1}, \quad l=l_{k}+1, l_{k}+2, \ldots, i_{k+1}-i_{k}-1$.

From (32), we see that $\Delta_{i_{k}-1} \rightarrow 0$ as $k \rightarrow \infty$. This fact combined with (36) and (38) implies that

$$
\lim _{k \rightarrow \infty, k \in J} \Delta_{k}=0 .
$$

Hence, it follows from (32) and (39) that (35) holds.

We now prove the theorem. From the Taylor expansion of $f(x), \mathrm{A}_{2},\left\|d_{k}\right\| \leqslant \Delta_{k}$, and (35), we obtain

$$
\begin{aligned}
\mid f_{k} & -f\left(x_{k}+d_{k}\right)-\left(\phi_{k}(0)-\phi_{k}\left(d_{k}\right)\right) \mid \\
& =\left|\frac{1}{2} d_{k}^{\mathrm{T}} B_{k} d_{k}-\int_{0}^{1}\left[g\left(x_{k}+\xi d_{k}\right)-g_{k}\right]^{\mathrm{T}} d_{k} \mathrm{~d} \xi\right| \\
& \leqslant O\left(\Delta_{k}^{2}\left\|B_{k}\right\|\right)+o\left(\Delta_{k}\right) .
\end{aligned}
$$

By (11), (31) and (40), it follows that

$$
\left|\frac{f_{k}-f\left(x_{k}+d_{k}\right)}{\phi_{k}(0)-\phi_{k}\left(d_{k}\right)}-1\right| \leqslant \frac{O\left(\Delta_{k}^{2}\left\|B_{k}\right\|\right)+o\left(\Delta_{k}\right)}{\tau \epsilon \min \left\{\Delta_{k}, \epsilon /\left\|B_{k}\right\|\right\}} .
$$

Combining the above inequality, (35) and $A_{3}$, we deduce that

$$
\lim _{k \rightarrow \infty} \frac{f_{k}-f\left(x_{k}+d_{k}\right)}{\phi_{k}(0)-\phi_{k}\left(d_{k}\right)}=1 .
$$

It follows from (12) and (15) that

$$
\rho_{k}=\frac{D_{k}-f\left(x_{k}+d_{k}\right)}{\phi_{k}(0)-\phi_{k}\left(d_{k}\right)} \geqslant \frac{f_{k}-f\left(x_{k}+d_{k}\right)}{\phi_{k}(0)-\phi_{k}\left(d_{k}\right)} .
$$

Thus for $k$ large enough, according to $\mu \in(0,1)$, (41) and (42), we have that

$$
\rho_{k} \geqslant \mu \text {. }
$$

From Step 5 of Algorithm 1, we know that $\Delta_{k+1} \geqslant$ $\Delta_{k}$ holds for sufficiently large $k$, which contradicts (35).

\section{LOCAL SUPERLINEAR CONVERGENCE}

In this section, we analyse the superlinear convergence of Algorithm 1 under suitable conditions. First we present the following assumptions.

$\left(\mathrm{A}_{4}\right) f(x)$ is twice continuously differentiable.

$\left(A_{5}\right)$ The matrix $B_{k}$ is invertible, $\left\|B_{k}^{-1} g_{k}\right\| \leqslant \Delta_{k}$, and Algorithm 1 chooses the step $d_{k}=-B_{k}^{-1} g_{k}$ for all $k$.
Theorem 2 Suppose that $\mathrm{A}_{1}, \mathrm{~A}_{3}, \mathrm{~A}_{4}, \mathrm{~A}_{5}$ hold. Suppose that the sequence $\left\{x_{k}\right\}$ is generated by Algorithm 1 and converges to a point $x^{*}$, where $\nabla^{2} f\left(x^{*}\right)$ is positive definite and $\nabla^{2} f(x)$ is Lipschitz continuous on a neighbourhood of $x^{*}$. If

$$
\lim _{k \rightarrow \infty} \frac{\left\|\left(B_{k}-\nabla^{2} f\left(x_{k}\right)\right) d_{k}\right\|}{\left\|d_{k}\right\|}=0,
$$

then the sequence $\left\{x_{k}\right\}$ converges to $x^{*}$ superlinearly.

Proof: According to $\mathrm{A}_{2}$ and Theorem 1, we know that there exists a constant $L_{1}>0$ such that

$$
\left\|g\left(x_{k}\right)-g\left(x^{*}\right)\right\| \leqslant L_{1}\left\|x_{k}-x^{*}\right\|,
$$

and the sequence $\left\{x_{k}\right\}$ is generated by Algorithm 1 and converges to a point $x^{*}$. It follows that

$$
\lim _{k \rightarrow \infty}\left\|g\left(x_{k}\right)-g\left(x^{*}\right)\right\| \leqslant 0 .
$$

This implies that

$$
\lim _{k \rightarrow \infty}\left\|g_{k}\right\|=\left\|g\left(x^{*}\right)\right\|=0 .
$$

Then it means that $x^{*}$ is a strict local minimizer. Suppose that $\Omega=\left\{x \mid\left\|x-x^{*}\right\| \leqslant \Delta\right\}$, where $\Delta>0$ is a sufficiently small constant such that $x_{k} \in \Omega$ for all $k \geqslant k_{0}$, where $k_{0}$ is a positive integer. From $\mathrm{A}_{4}$, we know that there exist two positive constants $m$ and $M$, such that

$$
m\|d\|^{2} \leqslant d^{\mathrm{T}} \nabla^{2} f(x) d \leqslant M\|d\|^{2}, \quad \forall d \in \mathbb{R}^{n},
$$

for all $x \in \Omega$. For sufficiently large $k>k_{0}$, from (2), it follows that

$\phi_{k}(0)-\phi_{k}\left(d_{k}\right)=\frac{1}{2} d_{k}^{\mathrm{T}}\left(B_{k}-\nabla^{2} f\left(x_{k}\right)\right) d_{k}+\frac{1}{2} d_{k}^{\mathrm{T}} \nabla^{2} f\left(x_{k}\right) d_{k}$.

From $\mathrm{A}_{3}$ we have that $d_{k} \rightarrow 0$ as $k \rightarrow \infty$. Combining $d_{k} \rightarrow 0$, (43), (45), and (46), we know that

$$
\lim _{k \rightarrow \infty} \frac{\phi_{k}(0)-\phi_{k}\left(d_{k}\right)}{\left\|d_{k}\right\|^{2}} \leqslant M
$$

This implies that

$$
\phi_{k}(0)-\phi_{k}\left(d_{k}\right)=O\left(\left\|d_{k}\right\|^{2}\right) .
$$

By the mean-value theorem, it follows that

$$
\begin{aligned}
f_{k}-f\left(x_{k}+d_{k}\right)-\left(\phi_{k}(0)-\phi_{k}\left(d_{k}\right)\right) & \\
=\frac{1}{2} d_{k}^{\mathrm{T}}\left(\nabla^{2} f\left(x_{k}\right)\right. & \left.-\nabla^{2} f\left(x_{k}+\xi d_{k}\right)\right) d_{k} \\
+ & \frac{1}{2} d_{k}^{\mathrm{T}}\left(B_{k}-\nabla^{2} f\left(x_{k}\right)\right) d_{k},
\end{aligned}
$$


where $\xi \in(0,1)$. For large enough $k$, from the Lipschitz continuity of $\nabla^{2} f(x)$, (43) and $d_{k} \rightarrow 0$, it follows that

$$
f_{k}-f\left(x_{k}+d_{k}\right)-\left(\phi_{k}(0)-\phi_{k}\left(d_{k}\right)\right)=o\left(\left\|d_{k}\right\|^{2}\right) .
$$

Combining (47) and (48), we know that

$$
\begin{aligned}
& \left|\frac{f_{k}-f\left(x_{k}+d_{k}\right)}{\phi_{k}(0)-\phi_{k}\left(d_{k}\right)}-1\right| \\
& \quad=\left|\frac{f_{k}-f\left(x_{k}+d_{k}\right)-\phi_{k}(0)+\phi_{k}\left(d_{k}\right)}{\phi_{k}(0)-\phi_{k}\left(d_{k}\right)}-1\right| \\
& \quad \leqslant \frac{o\left(\left\|d_{k}\right\|^{2}\right)}{O\left(\left\|d_{k}\right\|^{2}\right)}
\end{aligned}
$$

By (49), we know that (41) holds. Combining (41) and (42) we know that $\rho_{k} \geqslant \mu$ for sufficiently large $k$. Hence Algorithm 1 reduces to the standard quasiNewton method when $k$ is sufficiently large and we can obtain the superlinear convergence result by using the standard results of the quasi-Newton method.

\section{COMPUTATIONAL EXPERIMENTS}

In this section, we provide some preliminary numerical experiments to show the performance of our proposed algorithm. The new nonmonotone trust region algorithm is denoted by NNTR from now on. In Algorithm 1, the parameter $\eta$ has a wide scope. If we take $\eta=0$, then we can obtain the usual trust region methods described in Ref. 2 (denoted by UTR). We also compare Algorithm 1 with the nonmonotone trust region (NTR) method proposed by Mo et $\mathrm{al}^{10}$.

The mentioned algorithms were coded in MATLAB 7.1. All numerical computation were conducted using an Intel Core 2 Duo CPU $2.20 \mathrm{GHz}$ computer with $2 \mathrm{~GB}$ of RAM. For Algorithm 1 we used $\Delta_{0}=2$, $\mu=0.25, c_{1}=0.25, c_{2}=1.25$.

In all tests the maximum number of iterations is 300 , and the termination condition is $\left\|g_{k}\right\| \leqslant 10^{-6}$. In all algorithms, $B_{k}$ is updated by the following BFGS formula:

$$
B_{k+1}=B_{k}+\frac{B_{k} s_{k} s_{k}^{\mathrm{T}} B_{k}}{s_{k}^{\mathrm{T}} B_{k} s_{k}}+\frac{y_{k}^{*}\left(y_{k}^{*}\right)^{\mathrm{T}}}{\left(y_{k}^{*}\right)^{\mathrm{T}} s_{k}},
$$

where

$$
y_{k}^{*}=\frac{y_{k}^{\mathrm{T}} s_{k}}{\left|y_{k}^{\mathrm{T}} s_{k}\right|} y_{k}, \quad s_{k}=x_{k+1}-x_{k}, \quad y_{k}=g_{k+1}-g_{k} .
$$

For each test, we choose the initial matrix $B_{0}=\left|f_{0}\right| E$, where $E$ is the unit matrix.
Table 1 Numerical results for Example 1.

\begin{tabular}{cccccc}
\hline Dim & & \multicolumn{3}{c}{ NNTR $(\eta=0.2)$} \\
& Iter & FV & TCPU & NF & NG \\
\hline 32 & 44 & $2.54 \times 10^{-16}$ & 0.0559 & 89 & 84 \\
64 & 46 & $4.99 \times 10^{-17}$ & 0.0891 & 93 & 90 \\
128 & 42 & $1.64 \times 10^{-16}$ & 0.1874 & 85 & 83 \\
256 & 47 & $3.01 \times 10^{-16}$ & 0.7310 & 95 & 93 \\
512 & 45 & $1.65 \times 10^{-19}$ & 3.4084 & 91 & 91 \\
\hline
\end{tabular}

Iter = number of iterations; Dim = number of dimensions; FV = final value of $f\left(x_{k}\right)$; TCPU $=$ CPU time (s); number of function evaluations; number of gradient evaluations

Table 2 Numerical results for Example 2.

\begin{tabular}{cccccc}
\hline Dim & \multicolumn{5}{c}{ NNTR $(\eta=0.2)$} \\
& Iter & FV & TCPU & NF & NG \\
\hline 32 & 50 & $2.60 \times 10^{-11}$ & 0.0617 & 101 & 101 \\
64 & 50 & $5.44 \times 10^{-10}$ & 0.0766 & 101 & 101 \\
128 & 62 & $4.86 \times 10^{-13}$ & 0.2241 & 125 & 125 \\
256 & 62 & $1.43 \times 10^{-10}$ & 0.9593 & 125 & 125 \\
512 & 68 & $1.24 \times 10^{-9}$ & 5.0646 & 137 & 137 \\
\hline
\end{tabular}

Example 1 Extended Rosenbrock function. The test function is the 21st example of Ref. 11. Let

$$
f(x)=\sum_{i=1}^{n / 2}\left[100\left(x_{2 i}-x_{2 i-1}^{2}\right)^{2}+\left(1-x_{2 i-1}\right)^{2}\right] .
$$

The minimum of the problem is $f(\min )=0$. The standard starting point is $x_{0}=(-1.2,1, \ldots,-1.2,1)$.

Example 2 Extended Powell singular function. The test function is the 22nd example of Ref. 11. Let

$$
\begin{aligned}
f(x)= & \sum_{i=1}^{n / 4}\left[\left(x_{4 i-1}+10 x_{4 i-2}\right)^{2}+5\left(x_{4 i-2}-x_{4 i}\right)^{2}\right. \\
& \left.+\left(x_{4 i-2}-2 x_{4 i-1}\right)^{2}+10\left(x_{4 i-3}-x_{4 i}\right)^{4}\right] .
\end{aligned}
$$

The minimum of the problem is $f_{\min }=$ 0 . The standard starting point is $x_{0}=$ $(3,-1,0,1, \ldots, 3,-1,0,1)$.

Example 3 Extended Dixon test function. The test function is problem 4.5 of Ref. 12. Let

$$
\begin{aligned}
f(x)=\sum_{i=1}^{n / 10}\left[\left(1-x_{10 i-9}\right)^{2}\right. & +\left(1-x_{10 i}\right)^{2} \\
& \left.+\sum_{j=10 i-9}^{10 i-1}\left(x_{j}^{2}-x_{j+1}\right)^{2}\right] .
\end{aligned}
$$


Table 3 Numerical results for Example 3.

\begin{tabular}{cccccc}
\hline Dim & & \multicolumn{3}{c}{ NNTR $(\eta=0.2)$} \\
& Iter & FV & TCPU & NF & NG \\
\hline 32 & 80 & $7.40 \times 10^{-16}$ & 0.0475 & 161 & 160 \\
64 & 85 & $4.38 \times 10^{-16}$ & 0.1415 & 171 & 171 \\
128 & 106 & $1.09 \times 10^{-15}$ & 0.3623 & 213 & 211 \\
256 & 114 & $1.87 \times 10^{-16}$ & 1.8159 & 229 & 229 \\
512 & 130 & $1.38 \times 10^{-15}$ & 9.9293 & 261 & 261 \\
\hline
\end{tabular}

Table 4 Numerical results for Example 4.

\begin{tabular}{cccccc}
\hline Dim & & \multicolumn{3}{c}{ NNTR $(\eta=0.2)$} \\
& Iter & FV & TCPU & NF & NG \\
\hline 32 & 33 & $4.38 \times 10^{-16}$ & 0.0182 & 67 & 67 \\
64 & 28 & $7.47 \times 10^{-15}$ & 0.0724 & 57 & 57 \\
128 & 37 & $8.04 \times 10^{-15}$ & 0.1233 & 75 & 75 \\
256 & 55 & $1.01 \times 10^{-14}$ & 0.8374 & 111 & 111 \\
512 & 81 & $8.00 \times 10^{-15}$ & 5.9847 & 163 & 163 \\
\hline
\end{tabular}

The minimum of the problem is $f(\min )=0$. The standard starting point is $x_{0}=(-2,-2, \ldots,-2,-2)$.

Example 4 Broyden tridiagonal function. The test function is the 30th example of Ref. 11. Let

$$
f(x)=\sum_{i=1}^{n}\left[\left(3-2 x_{i}\right) x_{i}-x_{i-1}-2 x_{i+1}+1\right]^{2} .
$$

The minimum of the problem is $f(\min )=0$. The standard starting point is $x_{0}=(-1,-1, \ldots,-1,-1)$.

In tables 1-4 we give some test results about five large scale unconstrained optimization problems to show whether the parameter $\eta$ has an impact on Algorithm 1. We test the five problems with two cases. The dimensions of the problems are chosen from 32-512.

\section{CONCLUSIONS}

In this paper, we proposed a new nonmonotone trust region algorithm based on the nonmonotone line search proposed by $\mathrm{Gu}$ and $\mathrm{Mo}^{9}$. Theoretical analysis shows that the new algorithm inherits the global convergence of the traditional trust region method. Under suitable conditions the superlinear convergence of the algorithm is proved. Preliminary numerical experiments indicate that our algorithm is quite effective for large scale unconstrained optimization problems.

Acknowledgements: This work was supported by National Social Science Foundation of China (Grant No. 16BKS132). The authors thank the anonymous referees for their constructive comments and suggestions.

\section{REFERENCES}

1. Gertz EM (1999) Combination Trust-region Line Search Methods for Unconstrained Optimization, Univ of California, San Diego.

2. Deng NY, Xiao Y, Zhou FJ (1993) Nonmonotic trust region algorithm. J Optim Theor Appl 76, 259-85.

3. Grippo L, Lampariello F, Lucidi S (1986) A nonmonotone line search technique for Newton's method. SIAM J Numer Anal 23, 707-16.

4. Sun WY, Han JY, Sun J (2002) On the global convergence of nonmonotone descent methods. $J$ Comput Appl Math 146, 89-98.

5. Grippo L, Sciandrone M (2002) Nonmonotone globalization techniques for the Barzilai-Borwein gradient method. Comput Optim Appl 23, 143-69.

6. Dai YH (2002) On the nonmonotone line search. J Optim Theor Appl 112, 315-30.

7. Toint PL (1996) An assessment of non-monotone line search techniques for unconstrained optimization. SIAM J Sci Stat Comput 17, 725iijo 39 .

8. Zhang H, Hager WW (2004) A nonmonotone line search technique and its application to unconstrained optimization. SIAM J Optim 14, 1043-56.

9. Gu NZ, Mo JT (2008) Incorporating nonmonotone strategies into the trust region method for unconstrained optimization. Appl Math Comput 55, 2158-72.

10. Mo JT, Liu CY, Yan SC (2007) A nonmonotone trust region method based on nonincreasing technique of weighted average of the successive function values. J Comput Appl Math 209, 97-108.

11. More JJ, Garbow BS, Hillstrome KE (1981) Testing unconstrained optimization software. ACM Trans Math Software 7, 17-41.

12. Touati-Ahmed D, Storey C (1990) Efficient hybrid conjugate gradient techniques. J Optim Theor Appl 64, 379-97. 